\title{
Comment on "From geodesics of the multipole solutions to the perturbed Kepler problem"
}

\author{
L. Fernández-Jambrina* \\ Matemática Aplicada, E.T.S.I. Navales, Universidad Politécnica de Madrid, Arco de la Victoria s/n, E-28040 Madrid, Spain
}

\begin{abstract}
In this Comment we explain the discrepancies mentioned by the authors between their results and ours about the influence of the gravitational quadrupole moment in the perturbative calculation of corrections to the precession of the periastron of quasielliptical Keplerian equatorial orbits around a point mass. The discrepancy appears to be a consequence of two different calculations of the angular momentum of the orbits.
\end{abstract}

In [1] the authors make use of their static and axisymmetric solution of Einstein's vacuum equations with a finite number of multipole moments [2-4] in a system of coordinates adapted to multipole symmetry to derive a Binet equation for orbits in the equatorial plane and relate it to the classical Keplerian problem. This allows them to write down the relativistic corrections to Newtonian elliptical orbits in terms of multipole moments of the source of the gravitational field:

$$
\frac{d^{2} u}{d \varphi^{2}}+u=\frac{M}{J^{2}}+3 M u^{2}-\frac{1}{J^{2}} \frac{d}{d u} V_{p}^{R M M}(u)
$$

where $u$ is the inverse of the radial coordinate, $M$ is the central mass, $J$ is the angular momentum of the orbit, and $V_{p}^{R M M}(u)$ is a generalized gravitational potential which encloses the perturbations due to the gravitational quadrupole moment $Q$ after substracting the Schwarzschild and centrifugal terms. We have taken the gravitational constant $G$ and the mass of the orbiting test particle $m$ equal to one.

The authors obtain a result for the angle precessed by the periastron in a revolution around the quasielliptical orbit:

$$
\Delta \phi=6 \pi\left\{\zeta+\zeta^{2} \frac{Q}{M^{3}}\left(-\frac{1}{2}+3 \frac{M}{a}\right)\right\}
$$

where $a$ is the semimajor axis of the unperturbed orbital ellipse of eccentricity $e$ and, hence, of angular momentum $J= \pm \sqrt{a\left(1-e^{2}\right) M}$ and energy $E=-M / 2 a$. A dimensionless small parameter $\zeta=M^{2} / J^{2}$ has been introduced.

In [5] a different approach was followed to a similar purpose. Starting with the general metric for a stationary axially symmetric vacuum spacetime,

$$
\begin{aligned}
d s^{2}= & -f(d t-A d \phi)^{2} \\
& +\frac{e^{2 \gamma}\left(d r^{2}+r^{2} d \theta^{2}\right)+r^{2} \sin ^{2} \theta d \phi^{2}}{f},
\end{aligned}
$$

where $t$ and $\phi$ are the coordinates associated with the isometries of the spacetime and the functions $f, A$, and $\gamma$ depend only on the coordinates $r$ and $\theta$, a Binet equation is

\footnotetext{
*leonardo.fernandez@upm.es; http://dcain.etsin.upm.es/ilfj.htm
}

written for $U=1 / r$ along timelike geodesics in the spacetime:

$$
U_{\phi}^{2}=e^{-2 \gamma}\left\{\frac{E^{2}-f}{f^{2}(J-E A)^{2}}-U^{2}\right\}
$$

where $E$ and $J$, respectively, energy and angular momentum per unit of mass, are the conserved quantities of geodesic motion associated to the isometries of the spacetime:

$$
E=f(\dot{t}-A \dot{\phi}), \quad J=f A(\dot{t}-A \dot{\phi})+\frac{r^{2}}{f} \dot{\phi},
$$

and the dot means derivation with respect to proper time along the geodesic.

This Binet equation arises from the normalization condition of the velocity $v=(\dot{t}, \dot{r}, \dot{\theta}, \dot{\phi})$ of geodesics parametrized by proper time, $v \cdot v=-1$,

$$
-1=-f(\dot{t}-A \dot{\phi})^{2}+\frac{e^{2 \gamma} \dot{r}^{2}+r^{2} \dot{\phi}^{2}}{f},
$$

and imposing the existence of conserved quantities in order to remove the derivatives $\dot{\phi}$ and $\dot{t}$. Binet's equation (4) is obtained dividing by $\dot{\phi}^{2}$ and thereby eliminating the dependence on proper time.

In order to compare our results with [1], we take $A(r, \theta)=0$ in order to consider only static spacetimes.

This equation is solved perturbatively [5] in powers of a small dimensionless parameter $\epsilon=M / J$ and a change of variable $\psi=\omega \phi$, which allows us to get rid of secular terms in the perturbation scheme. This frequence $\omega$ is responsible for the precessed angle,

$$
\frac{\Delta \phi}{\pi}=\frac{1}{\omega}-1 \simeq 6 \epsilon^{2}+\left(\frac{105}{2}+15 E_{0}-\frac{3 Q}{M^{3}}\right) \epsilon^{4},
$$

whereas the energy is also expanded

$$
E \simeq 1+E_{0} \epsilon^{2}+\left(-6-10 E_{0}-\frac{E_{0}^{2}}{2}\right) \epsilon^{4},
$$

in powers of $\epsilon$. The term $E_{0}$ is related therefore to the classical orbit. 
The discrepancy mentioned in [1] arises on identifying the small parameters in both expansions by means of $\zeta=\epsilon^{2}$. The term $3 M / a$ in (2) appears to be missing in (7).

The explanation is simple and is due precisely to the previous identification. The authors assign the classical Keplerian values $E_{c}=-M / 2 a, J_{c}^{2}=a\left(1-e^{2}\right) M$ to the energy and momentum of the elliptical orbit. However, in their calculations $E$ and $J$ are also the conserved quantities of geodesic motion, and hence $J$ has the same meaning in both notations, though the value $J^{2} \simeq a\left(1-e^{2}\right) M$ should enclose the contribution of the quadrupolar moment.

Since we are interested in the first correction to the angular momentum, we perform just the classical calculation.

In classical mechanics the orbit of a particle moving under a central force of potential $V(r)$ has two conserved quantities, the angular momentum $J$ and the energy per unit of mass $E$ :

$$
J=r^{2} \dot{\phi}, \quad E=\frac{\dot{r}^{2}}{2}+\frac{r^{2} \dot{\phi}^{2}}{2}+V(r)=\frac{\dot{r}^{2}}{2}+\frac{J^{2}}{2 r^{2}}+V(r),
$$

in spherical coordinates $(r, \theta, \phi)$.

The gravitational potential due to a central mass $M$ and a quadrupole $M$ is not central:

$$
V(r, \theta)=-\frac{M}{r}+\frac{Q\left(3 \cos ^{2} \theta-1\right)}{r^{3}},
$$

but considering just orbits in the equatorial plane, it acts as if it were central with $V(r)=-M / r-Q / r^{3}$.

For simplicity we consider circular orbits of radius $r=a$. It is clear that they cannot be used for measuring precessed angles, but still they provide an easy computation of (2) and (7). They are located at extrema of the effective potential

$$
V_{\mathrm{eff}}(r)=\frac{J^{2}}{2 r^{2}}-\frac{M}{r}-\frac{Q}{r^{3}}
$$

and so their radius is a solution of

$$
M a^{2}-J^{2} a+3 Q=0 .
$$

For $J^{4}>12 M Q$ there are two circular orbits, but we are interested in the exterior one,

$$
a=\frac{J^{2}+\sqrt{J^{4}-12 M Q}}{2 M} \simeq \frac{J^{2}}{M}-\frac{3 Q}{J^{2}},
$$

since it is the one that appears as a perturbation of the classical orbit for small $Q$.

The conserved quantities for these circular orbits,

$$
E=\frac{J^{2}}{2 a^{2}}-\frac{M}{a}-\frac{Q}{a^{3}}, \quad J^{2}=M a+\frac{3 Q}{a},
$$

are seen to have simple classical corrections due to the presence of the quadrupole moment.

In fact, if in (2) we include the quadrupolar correction to the Keplerian angular momentum, $J^{2}=J_{c}^{2}+3 Q / a$, the lowest order, the Schwarzschild term,

$$
\frac{6 \pi M^{2}}{J^{2}} \simeq \frac{6 \pi M^{2}}{J_{c}^{2}}\left(1-\frac{3 Q}{J_{c}^{2} a}\right)=6 \pi\left(\zeta-\frac{3 Q}{M^{2} a} \zeta^{2}\right)
$$

a counterterm appears that cancels out the last term in (2).

Hence we have shown that the apparent discrepancy between the formulas for the precession of the periastron of an equatorial orbit around a mass endowed with quadrupole moment calculated in $[1,5]$ is solved by including a first-order classical contribution to the Keplerian angular momentum due to the gravitational quadrupole moment in [1].
[1] J. L. Hernández-Pastora and J. Ospino, Phys. Rev. D 82, 104001 (2010).

[2] R. Geroch, J. Math. Phys. (N.Y.) 11, 2580 (1970).

[3] R. O. Hansen, J. Math. Phys. (N.Y.) 15, 46 (1974).
[4] C. Hoenselaers and Z. Perjes, Classical Quantum Gravity 7, 1819 (1990).

[5] L. Fernández-Jambrina and C. Hoenselaers, J. Math. Phys. (N.Y.) 42, 839 (2001). 